\title{
AVALIAÇÃO DO TEMPO DE DESCARGA DE FERTILIZANTES EM SILOS: ESTUDO EXPERIMENTAL E DE SIMULAÇÃO EMPREGANDO ELEMENTOS DISCRETOS
}

\author{
Y. A. MELO ${ }^{1}$, B. C. SILVÉRIO ${ }^{2}$, K. G. SANTOS ${ }^{1}$ e J. L. VIEIRA NETO ${ }^{1}$ \\ ${ }^{1}$ Universidade Federal Do Triângulo Mineiro, Departamento de Engenharia Química \\ ${ }^{2}$ Universidade Federal Do Triângulo Mineiro, Departamento de Engenharia de Alimentos \\ E-mail para contato: jose.neto@uftm.edu.br
}

\begin{abstract}
RESUMO - Silos são estruturas usadas no armazenamento de fertilizantes. Para predizer a dinâmica das partículas no interior dos mesmos podem ser empregados modelos computacionais. No estudo de escoamentos de sólidos o Método de Elementos Discretos, empregando abordagem Lagrangeana, exerce um papel importante, já que, considera a interação individual entre as partículas. No presente trabalho, realizou-se um estudo numérico para calibração de parâmetros de um destes modelos, o de Hertz-Mindlin, onde os tempos simulados foram comparados com o tempo de descarga experimental em várias condições de um planejamento composto central. Assim, considerando as simulações realizadas e as análises estatísticas, identificaram-se quais parâmetros influenciam no tempo de descarga definindo-se a melhor calibração.
\end{abstract}

\section{INTRODUÇÃO}

Os silos são empregados no armazenamento de fertilizantes a granel, pois, oferecem preservação da qualidade dos mesmos e segurança no processo. Assim, conhecer a dinâmica das partículas nesses equipamentos pode aperfeiçoar operações de transporte, descarga e armazenagem, bem como, evitar acidentes devido ao projeto inadequado. Os principais problemas que afetam a descarga de silos encontram-se relacionados à geometria, às propriedades do produto, ao modo o qual o produto é depositado no silo e ao tipo de fluxo durante a descarga. A agilidade na obtenção de resultados, a facilidade de acesso a programas prontos, e a variedade de propriedades que podem caracterizar o objeto de estudo, são pontos vantajosos do emprego de simulações computacionais para solução de problemas complexos.

\subsection{Objetivos}

Este trabalho tem como objetivo obter a melhor condição de calibração dos parâmetros do modelo de Hertz-Mindlin adotado nas simulações desenvolvidas no software EDEM Academic, bem como, a validação destes resultados numéricos confrontando com o tempo experimental de descarga de partículas de fertilizantes em um silo cônico-cilíndrico.

\section{MÉTODOS}

O Método de Elementos Discretos (DEM) desempenha importante papel no estudo do escoamento de sólidos. Empregando a abordagem Lagrangeana, esse método baseia-se na interação individual entre as partículas. Um estudo numérico para a calibração dos parâmetros do modelo Hertz-Mindlin foi então realizado no presente trabalho. As simulações foram executadas no software EDEM Academic com um passo no tempo equivalente a $20 \%$ do passo de tempo de Rayleigh e um tamanho de célula igual a 3Rmin. 


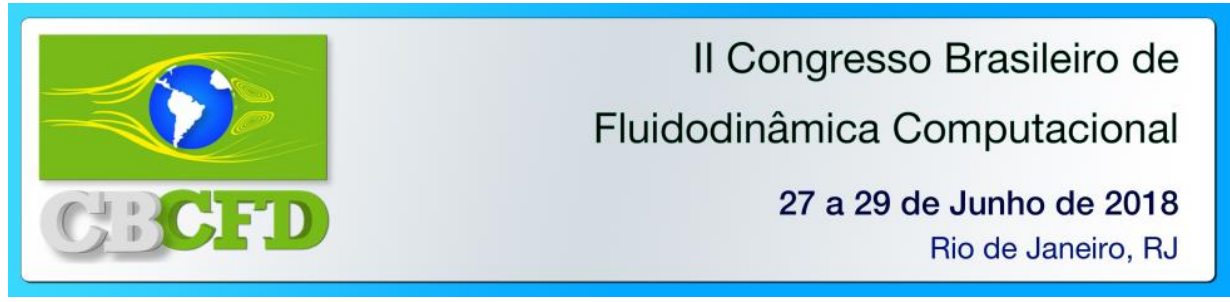

Na descarga do fertilizante foram previamente simulados 1,10016s (com saída do silo fechada) para empacotar os grãos. A partir da simulação de silo empacotado, os parâmetros do modelo de Hertz-Mindlin foram então alternados e as simulações de descarga (com saída inferior aberta) foram feitas para obter a melhor calibração.

Através de 27 simulações geradas, segundo um planejamento composto central (PCC), os tempos simulados foram comparados com o tempo de descarga experimental de fertilizantes em um silo. Os seguintes parâmetros foram avaliados: módulo de cisalhamento $(\mathrm{G})$, coeficiente de restituição $(\eta)$, coeficiente de atrito estático $\left(\mu_{s}\right)$ e coeficiente de atrito de rolamento $\left(\mu_{\text {rol }}\right)$, conforme a Tabela 1 apresentada a seguir.

\begin{tabular}{ccccccc}
\multicolumn{7}{c}{ Tabela 1 - Níveis das variáveis reais e codificadas empregadas no PCC } \\
\hline \multicolumn{7}{c}{ Níveis } \\
\hline Variáveis & $\begin{array}{c}\text { Variáveis } \\
\text { codificadas }\end{array}$ & $\mathbf{- 1 , 5 4 7}$ & $\mathbf{- 1}$ & $\mathbf{0}$ & $+\mathbf{1}$ & $+\mathbf{1 , 5 4 7}$ \\
\hline $\mathbf{G}(\mathbf{P a})$ & $\mathrm{X}_{1}$ & $1,36 \mathrm{E}+06$ & $3,00 \mathrm{E}+06$ & $6,00 \mathrm{E}+06$ & $9,00 \mathrm{E}+06$ & $1,06 \mathrm{E}+07$ \\
$\eta$ & $\mathrm{X}_{2}$ & 0,036 & 0,2 & 0,5 & 0,8 & 0,964 \\
$\mu_{s}$ & $\mathrm{X}_{3}$ & 0,0407 & 0,15 & 0,35 & 0,55 & 0,659 \\
$\mu_{r o l}$ & $\mathrm{X}_{4}$ & 0,0407 & 0,15 & 0,35 & 0,55 & 0,659
\end{tabular}

\subsection{Parâmetros do Modelo}

Coeficiente de restituição: Considerando que a resistência oferecida pelo ar pode ser desprezada, Aryaei et al. (2010), mostraram que o coeficiente de restituição $(\eta)$ pode ser dado pela Equação 1, sendo $h_{0}$ e $h_{1}$ a altura inicial e de rebote respectivamente.

$$
\eta=\sqrt{\frac{h_{1}}{h_{0}}}
$$

Coeficiente de atrito estático e cinético: Junior (2015) relaciona o coeficiente de atrito estático $\left(\mu_{s}\right)$ à força mínima necessária para iniciar o movimento, enquanto que o coeficiente de atrito cinético encontra-se relacionado à força mínima para manter o deslizamento.

Coeficiente de atrito de rolamento: Coeficiente de atrito de rolamento $\left(\mu_{\text {rol }}\right)$ é definido como a resistência causada ao movimento de rolamento de uma partícula esférica, onde a Lei de Coulomb pode ser utilizada.

Módulo de cisalhamento: $O$ módulo de cisalhamento, indicado $\operatorname{por} G$, é um parâmetro elástico definido para o esforço de cisalhamento como indicado na Equação 2, na qual $\tau$ é a tensão cisalhante e $\gamma$ indica a deformação elástica de cisalhamento do corpo de prova.

$$
G=\frac{\tau}{\gamma}
$$

\subsection{Materiais}

Um fertilizante de liberação lenta, conhecido como Osmocote 14-14-14 (importado dos EUA), geralmente usado por floricultores no cultivo de orquídeas foi empregado no presente trabalho. Ele foi escolhido por possuir algumas boas características para comparar a descarga experimental com as simuladas, tais como, partículas esféricas, não quebradiças, e não apresentar tendência de formar aglutinados. $\mathrm{O}$ fertilizante foi caracterizado e obteve-se um diâmetro médio $(\mathrm{dp})$ de $0,313 \mathrm{~cm}$, densidade $\left(\rho_{p}\right)$ de $1612 \mathrm{~kg} / \mathrm{m}^{3}$, e a porosidade $(\varepsilon)$ de $27 \%$. 


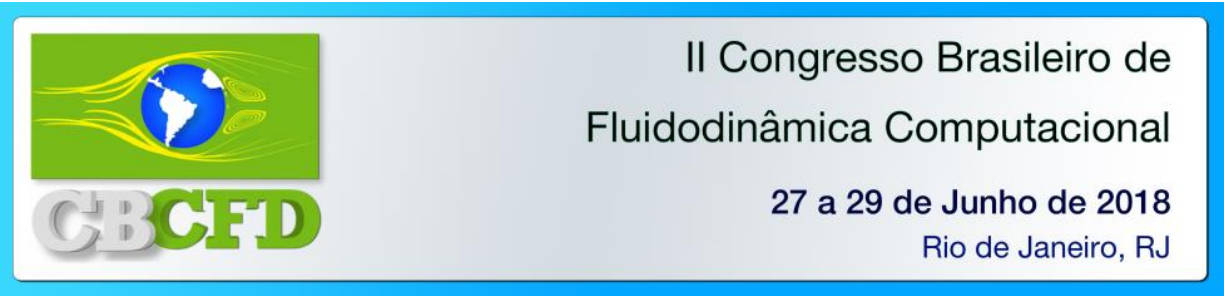

O silo foi disposto sobre uma balança digital e carregado com $0,305 \mathrm{~kg}$ de fertilizantes até que a parte cônica e somente essa, estivesse preenchida. A descarga das partículas foi filmada, de forma a possibilitar o acompanhamento da massa que deixava o silo em cada instante de tempo, bem como, o tempo total necessário para a descarga de todo o material particulado. Essa etapa deu-se em triplicata.

A Figura 1 mostra o fertilizante Osmocote 14-14-14 e o silo empregados neste estudo.

(a)

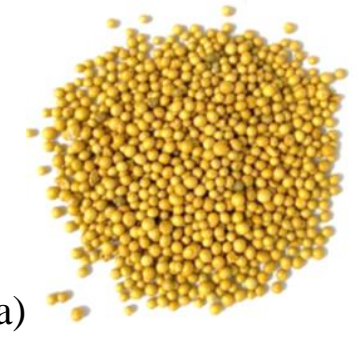

(b)

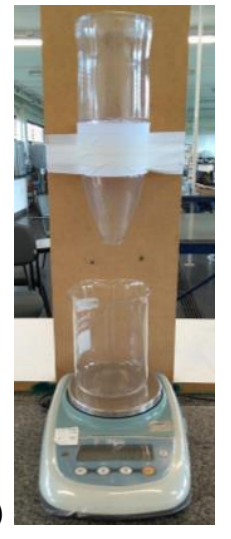

Figura 1 - Materiais: a) Fertilizante Osmocote, b) Silo utilizado para descarga experimental.

\section{RESULTADOS E DISCUSSÕES}

A Equação 3 representa o tempo de descarga em diferentes condições de parâmetros do modelo de contato. Nota-se uma maior influencia do atrito estático $\left(\mathrm{X}_{3}\right)$, seguida do atrito de rolamento $\left(\mathrm{X}_{4}\right)$. Há ainda efeitos quadráticos do atrito de rolamento e interações entre estes parâmetros. As superfícies de resposta na Figura 2 evidenciam esse resultado.

$$
t=3,5263+1,1227 X_{3}+0,2178 X_{4}-0,1739 X_{4}^{2}-0,0600 X_{2} X_{3}+0,2062 X_{3} X_{4}
$$

(a)

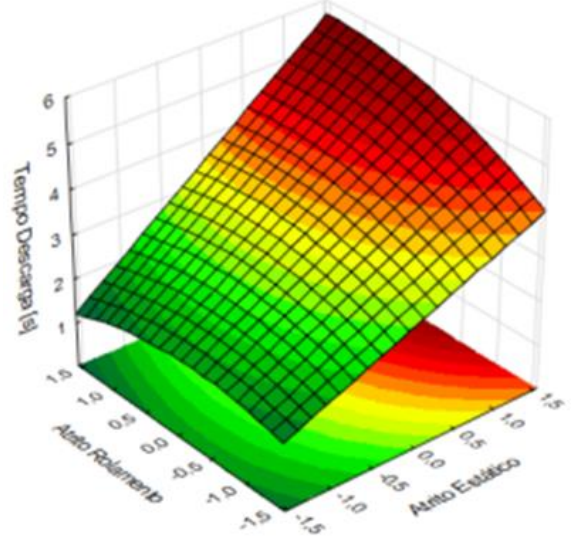

(b)

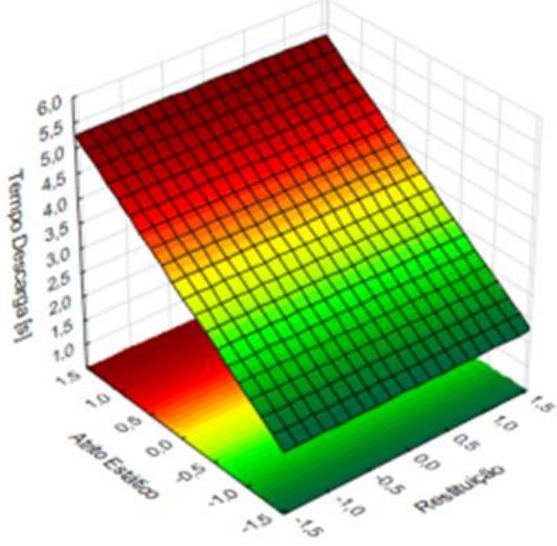

Figura 2 - Superfície de resposta para descarga do fertilizante em função dos coeficientes: (a) atrito de rolamento $\left(\mathrm{X}_{4}\right)$ e atrito estático $\left(\mathrm{X}_{3}\right)$, (b) atrito estático $\left(\mathrm{X}_{3}\right)$ e restituição $\left(\mathrm{X}_{2}\right)$

A partir dos resultados das simulações pode-se observar na Figura 3 que os tempos de descarga mais próximos do valor experimental $\left(\mathrm{t}_{\mathrm{exp}}=4,10 \mathrm{~s}\right)$ foram obtidos nas simulações do PCC 3,15, 11 e 7 (Figura 3c), em que o coeficiente de atrito estático está no nível +1 (com $\left.\mu_{s}=0,55\right)$ e o coeficiente de atrito de rolamento está no nível -1 (com $\mu_{\text {rol }}=0,15$ ), apresentando erros relativos entre 0,73 e $4,39 \%$ quando comparados ao valor experimental. 

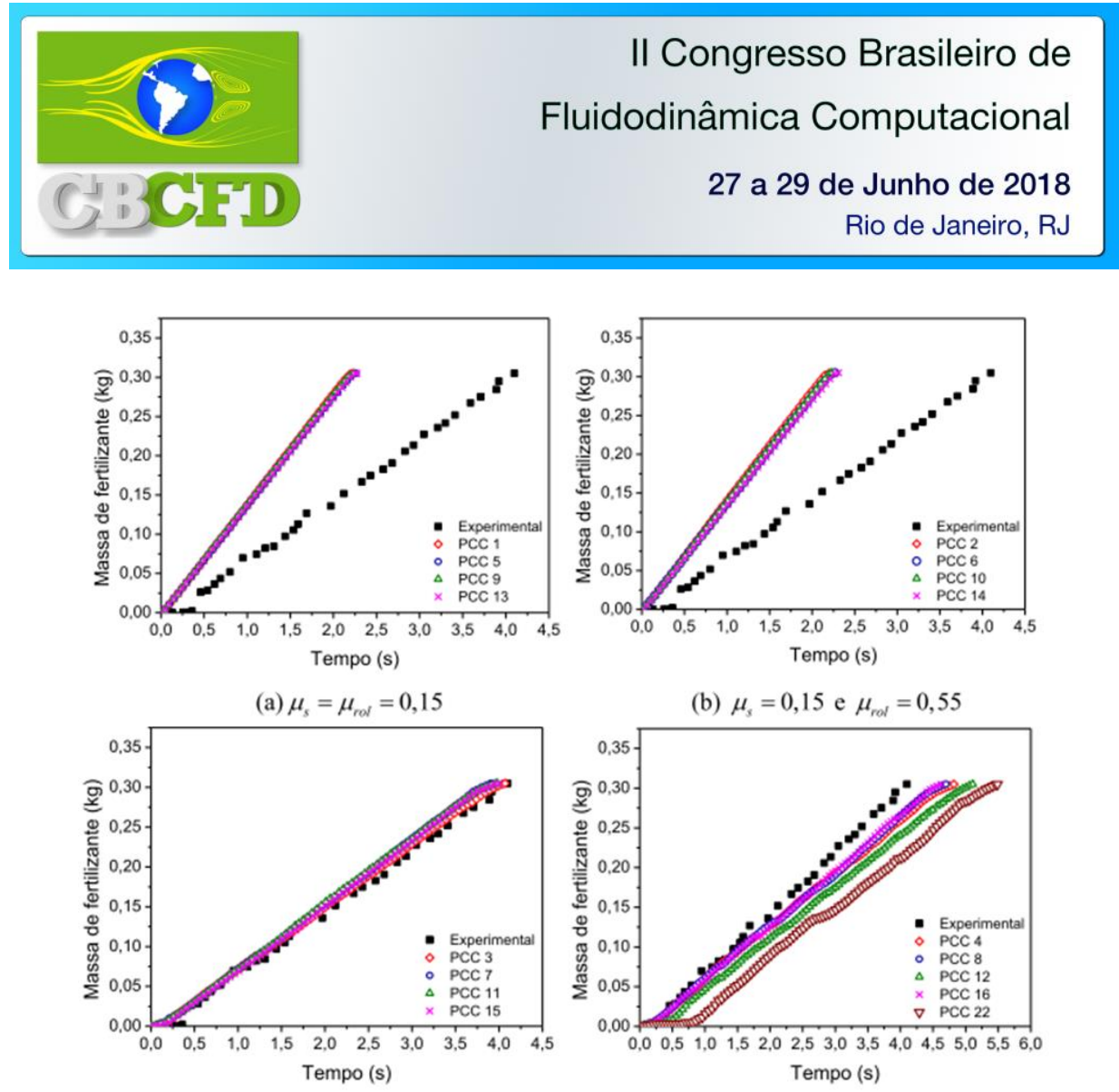

(c) $\mu_{s}=0,55$ e $\mu_{r o l}=0,15$

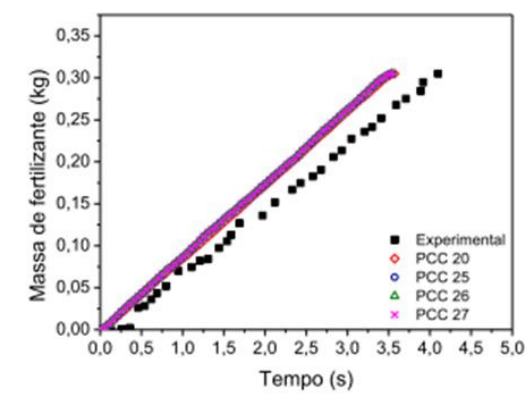

(d) $\mu_{s}=0,55$ e $\mu_{\text {rol }}=0,55$

(e) $\mu_{s}=0,35$ e $\mu_{\text {rot }}=0,35$

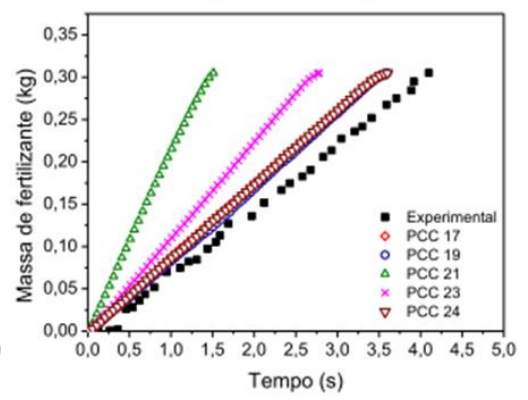

(f) Parâmetros nos níveis $+\alpha$ e $-\alpha$

Figura 3 - Comparação dos perfis de descarga de fertilizantes experimental e simulado.

\section{CONCLUSÕES}

Foi possível identificar, pelas simulações e análises estatísticas, os parâmetros para a melhor calibração da descarga do fertilizante Osmocote 14-14-14 em silo, com valores de módulo de cisalhamento $(\mathrm{G})$ de $3.10^{6} \mathrm{~Pa}$, coeficiente de restituição $(\eta)$ de 0,2 , coeficiente de atrito estático $\left(\mu_{\mathrm{s}}\right)$ de 0,55 e coeficiente de atrito de rolamento $\left(\mu_{\mathrm{rol}}\right)$ de 0,15 . Os coeficientes de atrito estático e de rolamento são os fatores que mais influenciam no tempo de descarga

\section{REFERÊNCIAS}

ARYAEI, A.; HASHEMNIA, K.; JAFARPUR, K. Experimental and numerical study of ball size effect on restitution coefficient in low velocity impacts. International Journal of Impact Engeneering, v. 37, n. 10, p. 1037-1044, 2010.

JUNIOR, M. M. Influência da topografia na força de atrito em papeis para embalagem. Tese

(Doutorado em Engenharia Química), UFSC, Florianópolis, 2015.

\section{AGRADECIMENTOS}

Os autores agradecem suporte do CNPq (460513/2014-1) e Fapemig (APQ-01139-14). 